\title{
3,4-二取代-3-(二氟甲基)吡唑类化合物的构建
}

\author{
曾俊良 ${ }^{*}, a$ 许志红 ${ }^{a}$ 马军安*,b \\ $\left({ }^{a}\right.$ 许昌学院化学化工学院 许昌 461000$)$ \\ ( ${ }^{b}$ 天津大学化学系 天津市分子光电科学重点实验室 天津化学化工协同创新中心 天津 300072)
}

\begin{abstract}
摘要 二氟甲基在活性分子中可以起到氢键供体、生物电子等排体、亲脂性调节等作用，在药物和农药分子的设计中 被广泛使用. 3,4-二取代-3-(二氟甲基)吡唑骨架的发现及应用是该领域的一个突出代表，研究表明含有这类骨架的分子 能够抑制琥珀酸脱氢酶(SDHIs), 具有作为杀菌剂的潜力, 目前市场上已有近十种农药分子含有 3,4-二取代-3-(二氟甲 基)吡唑结构单元，年销售额高达十几亿美元. 按照所用含氟砌块的不同种类，对近二十年来 3,4-二取代-3-(二氟甲基) 吡唑类化合物的合成方法进行总结和展望.
\end{abstract}

关键词 3,4-二取代-3-(二氟甲基)吡唑; 二氟甲基杂环化合物; 杀菌剂; 合成

\section{Construction of 3,4-Disubstituted-3-(difluoromethyl)pyrazoles}

\author{
Zeng, Junliang $^{*, a} \quad \mathrm{Xu}$, Zhihong $^{a} \quad$ Ma, Junan ${ }^{*, b}$ \\ ( ${ }^{a}$ School of Chemistry and Chemical Engineering, Xuchang University, Henan 461000) \\ ( ${ }^{b}$ Tianjin Key Laboratory of Molecular Optoelectronic Sciences, and Tianjin Collaborative Innovation Center of \\ Chemical Science \& Engineering, Department of Chemistry, Tianjin University, Tianjin 300072)
}

\begin{abstract}
The $\mathrm{CHF}_{2}$ moiety has been widely utilized in the design of pharmaceuticals and agrochemicals, because this group can act as hydrogen-bonding donor to improve the binding selectivity of biologically active compounds, as a bioisostere to substitute for methyl, methoxy, hydroxy, amino and thiol groups, and as a lipophilic regulator to improve the liposolubility of the active compounds. For example, 3-difluoromethylpyrazole scaffolds are present in many organic compounds that exhibit important biological activities. In this content, there are nearly ten kinds of pesticide molecules on the market that contain 3,4-disubstituted-3-(difluoromethyl)pyrazole units, with annual sales of up to one billion dollars. In this review, the methods of construction of 3,4-disubstituted 3-difluoromethylpyrazoles will been briefly summarized that have been reported so far. Four different strategies including using fluorinated reagents as substrates, difluoroacetic acid and its derivatives as fluorine building blocks, difluorodiazonium and others as fluorine building blocks will be introduced.
\end{abstract}

Keywords 3,4-disubstituted-3-difluoromethylpyrazoles; difluoromethyl heterocyclic compounds; fungicides; synthesis

含氟化合物因其特殊的氟效应在近几十年来受到 越来越多的关注. 其中含有二氟甲基 $\left(\mathrm{CHF}_{2}\right)$ 化合物的合 成研究备受青睐. 主要原因如下: (1) $\mathrm{CHF}_{2}$ 可以作为氢 键供体官能团. 氟原子的吸电子作用使得同碳氢原子具 有一定的活性(HB acidity: 0.035 vs 0.165 ), 可以作为弱 的氢键供体; (2) $\mathrm{CHF}_{2}$ 可以作为一些官能团的生物电子 等排体. 氟原子与氢原子大小较接近, $\mathrm{CHF}_{2}$ 可以替换活 性分子中的甲基、甲氧基、羟基、氨基、巯基等; (3) $\mathrm{CHF}_{2}$ 的引入可以调节分子的亲脂性. 例如 $\mathrm{CHF}_{2}$ 替换芳香烃 或烷烃分子中的甲基之后, 亲脂性减弱. 与之相反, $\mathrm{CHF}_{2}$ 替换分子中的羟基后, 亲脂性显著增加 $(\log P 1.67$ vs $\log P$ 3.02 $)^{[1]}$. 这些因素促使二氟甲基官能团在药物 和农药分子的设计中被广泛使用.

3,4-二取代-3-(二氟甲基)吡唑骨架的发现和应用是 二氟甲基官能团在农药分子设计中的一个重大突破. 这 一含氟活性杂环骨架在 1992 年由美国孟山都公司发现, 它能够抑制琥珀酸脱氢酶(SDHIs), 具有作为杀菌剂的 潜力 ${ }^{[2]}$, 但是随后的十年并没有新的成果或药物上市. 直到 2004 年, 巴斯夫(BASF SE)、先正达(Syngenta)、拜 耳农作物科学(Bayer)等公司先后发展一系列含有 3-(二 氟甲基)吡唑骨架的农药明星分子 ${ }^{[3]}$. 目前已上市的有: 巴斯夫研发的氟唑菌酰胺(年销售额约 4.1 亿美元), 先

*Corresponding authors. E-mail: junlzeng@tju.edu.cn; majun_an@tju.edu.cn

Received December 18, 2019 revised February 3, 2020; published online February 23, 2020.

Project supported by the Key Project of Education Department of Henan Province (Nos. 20A150039).

河南省高等学校重点科研(No. 20A150039)资助项目. 
正达的苯并烯氟菌唑(年销售额约 3 亿美元), 先正达的 氟唑环菌胺(年销售额约 3 亿美元), 拜耳作物科学研发 的杀菌剂联苯吡菌胺(年销售额约 1.7 亿美元) 等 ${ }^{[4]}$. 此外 还有不少公司正在积极开发新型含有 3-(二氟甲基)吡唑 结构单元的杀菌剂药物分子(图 1). 根据统计数据显示, 2016 年, 琥珀酸脱氢酶抑制剂(SDHIs)类杀菌剂的全球 销售额为 16.92 亿美元, 占 152.46 亿美元杀菌剂(包括非 作物用杀菌剂)市场的 $11.1 \%$. 其中以 3,4-二取代-3-二 氟甲基)吡唑骨架为母核结构的杀菌剂占据 SDHIs 类产 品市场的 $70 \%$ 以上 ${ }^{[5]}$. 由此可见, 发展廉价、高效的方 法构建 3,4-二取代-3-(二氟甲基)吡唑类化合物尤为重要.

\section{$13 ， 4-二$ 取代-3-(二氟甲基)吡唑类化合物的合 成}

由于市场应用取得的巨大成功, 3,4-二取代-3-(二氟 甲基)吡唑类化合物合成研究引起了众多的关注，相关 报道层出不穷. 本文按照所用含氟砌块种类的不同, 主 要从四个方面对 3,4-二取代-3-(二氟甲基)吡唑类化合物 的构建进行简单介绍.

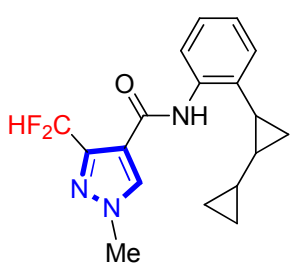

Sedaxane (Syngenta)

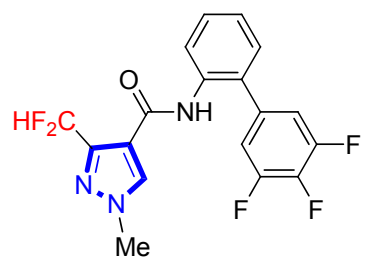

Fluxapyroxad (BASF SE)

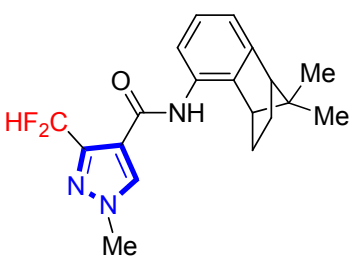

Isopyrazam (Syngenta)

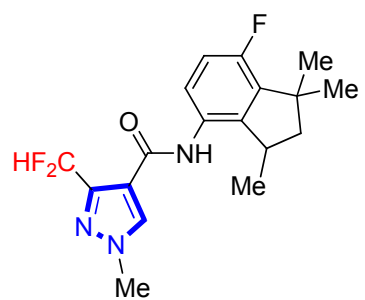

Fluindapyr (Isagro and FMC)

\section{1 以氟化试剂直接作为氟化底物}

利用氟化试剂在反应的后期通过取代或加成反应 引入氟元素是合成含氟化合物的一个重要且行之有效 的手段. 常见的氟化试剂有: 氟化钾、二乙胺基三氟化 硫(DAST)、氢氟酸、氢氟酸盐等. 2005 年, 拜尔生物 ${ }^{[6]}$ 利用二氯乙酰氯作为起始原料, 经过 $4 \sim 5$ 步反应制得 3,4-二取代-3-(二氯甲基)吡唑类化合物 4, 然后在氟化试 剂(如三乙胺三氢氟酸盐)作用下实现 3,4-二取代-3-(二 氟甲基)吡唑结构单元 5 的构建 (Schemes 1b, 1c). 该方法 虽然所用的起始原料二氯乙酰氯廉价易得，但是总收率 较低. 2008 年, 先正达公司 ${ }^{[7]}$ 对该路线进一步优化, 巧 妙地利用乙烯基醚作为底物，与二氯乙酰氯反应，得到 丙烯酮中间体，随后与甲基肼环化制得 3-(二氯甲基)吡 唑 2, 利用三乙胺氢氟酸盐通过亲核取代实现 3,4-二取 代-3-(二氟甲基)吡唑骨架的构建 (Scheme 1a). 虽然该方 法各步收率较高, 但是反应条件较为苛刻, 例如: 最后 的插羰反应, 要求严格无水无氧, 而且需要较高的温度 和压力. 此外, 上述这种合成方法在环化过程中不可避 免地产生 4,5-二取代的区域选择性异构体

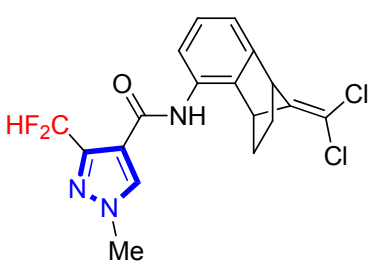

Benzovindiflupyr (Syngenta)

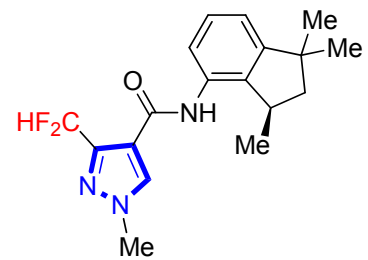

Inpyrfluxam (Sumitomo chemical)
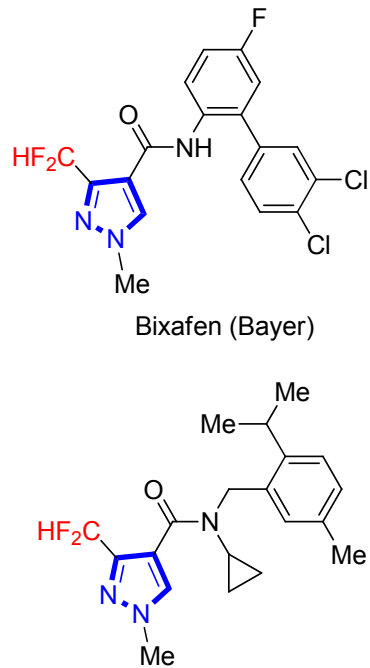

Isoflucypram (Bayer)
Pyrapropoyne (Nissan chemical industries)

图 1 含有 3-(二氟甲基)吡唑骨架的药物分子

Figure 1 Drug molecules containing 3- $\mathrm{CHF}_{2}$-pyrazole skeleton 


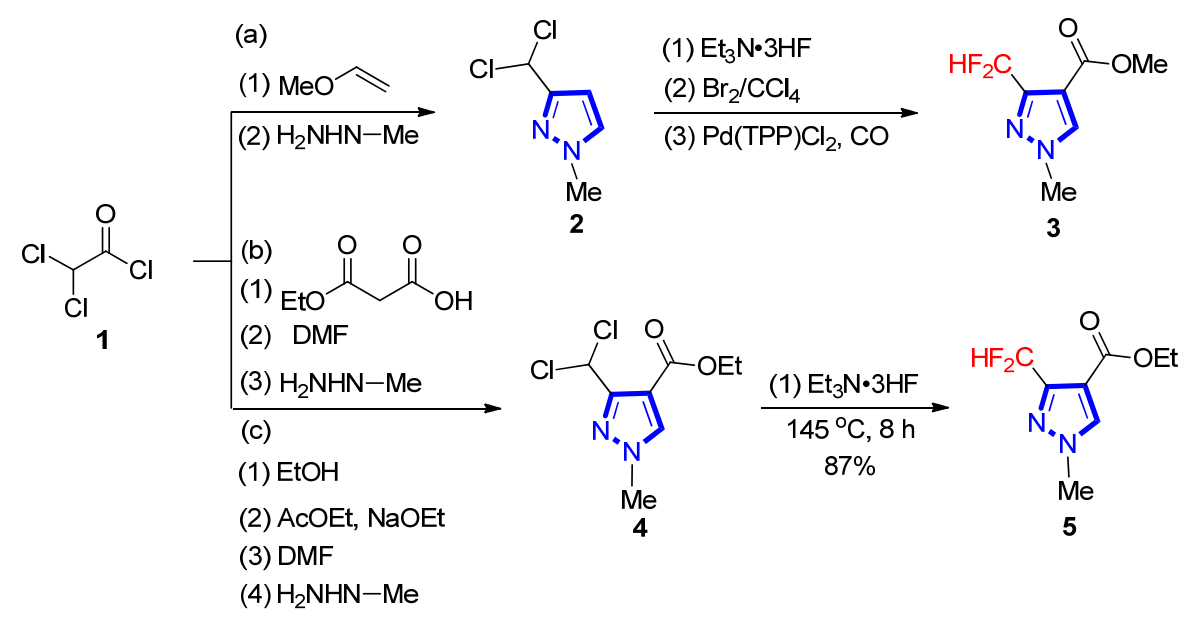

图式 1 以三乙胺三氢氟酸盐作为氟源合成 3-(二氟甲基)吡唑-4-羧酸酯类化合物

Scheme 1 Synthesis of 3- $\mathrm{CHF}_{2}$-pyrazole-4-carboxylate by using $\mathrm{Et}_{3} \mathrm{~N} \cdot 3 \mathrm{HF}$

2009 年, Kappe 小组 ${ }^{[8]}$ 对上述合成方法进行优化. 在最后氟化过程采用了微波反应法, 在极短的时间内实 现了 3-(二氯甲基)吡唑类化合物的脱氯氟化(Scheme 2). 该反应收率较高, 但对于 4-位连接有酯基取代基的 3-(二氯甲基)吡唑反应过程中有部分酯水解现象(比例 $10 \%)$. 此外该反应对温度和时间都需要严格控制, 温度 过高或过低、时间过长都会导致副反应的发生.<smiles></smiles>

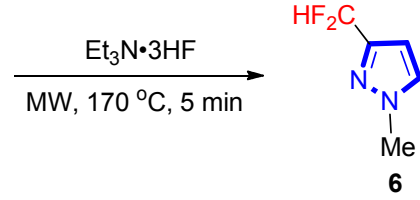

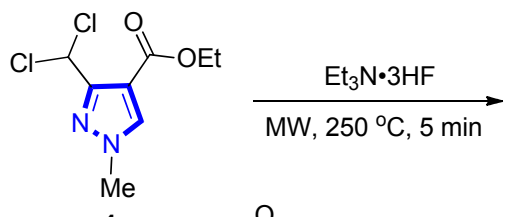

4<smiles>CCOC(=O)c1cn(N)nc1C(F)F</smiles><smiles>[Y6]n1cc(C(=O)O)c(C(F)F)n1</smiles>

图式 2 微波法实现 3-(二氟甲基)吡唑-4-羧酸酯的合成 Scheme 2 Synthesis of 3- $\mathrm{CHF}_{2}$-pyrazole-4-carboxylate by microwave irradiation

2011 年, Bolea 小组 ${ }^{[9]}$ 利用 $\beta$-酩酸酯类化合物制得单 取代吡唑类化合物 $\mathbf{8}$, 在二异丙基氨基锂(LDA)催化作 用下，选择性的实现了吡唑 3-位醛基的引入，制得吡唑 醛类化合物 9, 最后通过氟化试剂DAST, 以中等的收率 实现了 3,4-二取代-3-(二氟甲基)吡唑酰胺类化合物 10 的 合成(Scheme 3). 该反应虽然只有中等的收率, 但是巧 妙的通过官能团醛基的引入, 为 DAST 氟化提供了高的
位点选择性.

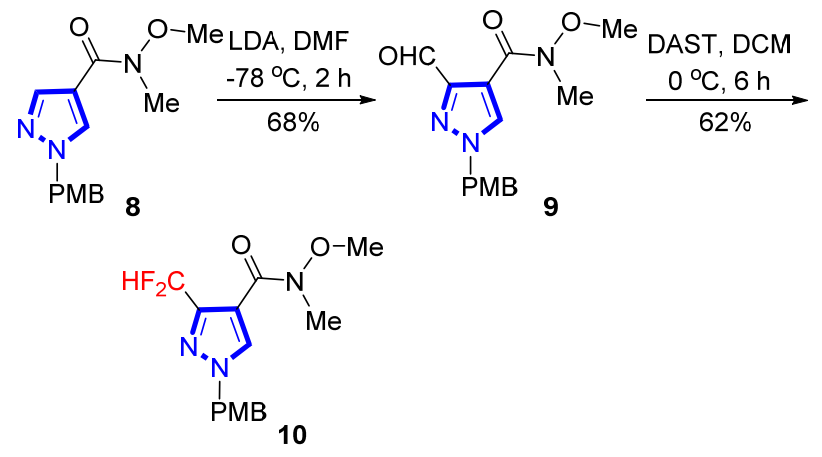

图式 3 DAST 用于 3-(二氟甲基)吡唑类化合物的合成 Scheme 3 Construction of 3-CHF 2 -pyrazoles by using DAST

2014 年, 焚小涁小组 ${ }^{[10]}$ 利用氰基烯胺类化合物 $\mathbf{1 1}$ 作为起始原料，与水合肼反应制得吡唑腈类化合物 $\mathbf{1 2}$, 随后在紫外光照条件下，与氯气通过自由基反应制得 3-(二氯甲基)吡唑. 接着在氟化试剂氟化氢、三乙胺三氢 氟酸盐、吡啶氟化氢络合液等作用下实现脱氯氟化, 制 得 3-(二氟甲基)吡唑腈类化合物 13. 最后通过甲基化、 水解反应得到 3,4-二取代-3-(二氟甲基)吡唑羒酸类化合 物 7 (Scheme 4). 该反应各步反应收率都达到 $90 \%$, 反 应效果较好, 显示了这条路线的优良合成应用价值.

2017 年, Maruoka 课题组 ${ }^{[11]}$ 使用二氟甲基乙酸碘苯 16 作为氟源, 与吡唑羒酸酯 15 在紫外光照条件下通过 自由基反应实现 3-(二氟甲基)吡唑-4-羧酸酯类化合物 3 的合成(Scheme 5). 该反应虽然收率较低，只有 $22 \%$ 的 核磁收率，但是成功地实现了缺电子芳杂环吡唑的直接 二氟甲基化, 具有较高的深入研究价值.

2018 年, 马军安小组 ${ }^{[12]}$ 在该课题组前期研究工 作 ${ }^{[13]}$ 的基础上，使用硝基烯烃和重氮乙腈直接构建出 3-(甲酰基)吡唑类化合物 19, 随后利用氟化剂 DAST 通 
<smiles>CC(=O)/C(=C/N(C)C)CN</smiles>

$\underset{\left(\text { (1) } \mathrm{HF}, \mathrm{TiCl}_{5}, 90 \%\right.}{\stackrel{\mathrm{h} v(285 \sim 365 \mathrm{~nm})}{\longrightarrow}} \stackrel{\mathrm{HF}_{2} \mathrm{C}}{\stackrel{\mathrm{Me}_{2} \mathrm{SO}_{4} \text {, toluene }}{100^{\circ} \mathrm{C}, 1 \mathrm{~h}, 95 \%}}$<smiles>Cn1cc(C#N)c(C(F)F)n1</smiles><smiles>[Y6]n1cc(C(=O)O)c(C(F)(F)F)n1</smiles>

图式 4 以 $\mathrm{HF}$ 作为氟源合成 3-(二氟甲基)吡唑-4-羧酸 Scheme 4 Construction of $3-\mathrm{CHF}_{2}-$ pyrazole- $4-\mathrm{CO}_{2} \mathrm{H}$ by using HF

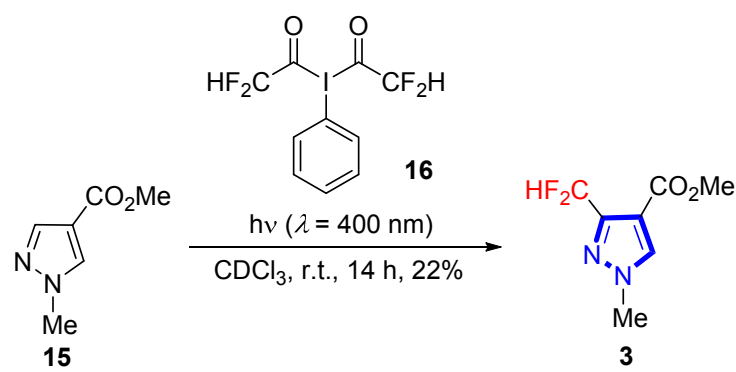

图式 5 3-(二氟甲基)吡唑-4-羧酸酯类化合物的合成 Scheme 5 Synthesis of 3-CHF 2 -pyrazole-4-carboxylate by direct difluoromethylation of pyrazole

过亲核加成反应实现了一系列 3-(二氟甲基)吡唑类化合 物 20 的合成. 该反应底物普适性较好, 收率高达 $95 \%$. 其中呋喃环取代的 3-(二氟甲基)吡唑类化合物 21 在高 碘酸钠和三氯化钉的作用下, 氧化开环得到 3,4-二取 代-3-(二氟甲基)吡唑-4-羧酸类化合物(Scheme 6). 该方 法路线简短、底物易得, 具有优良的合成应用价值.

\section{2 以二氟乙酸及其衍生物作为氟砌块}

二氟乙酸及其衍生物因其价廉易得且含有二氟甲 基官能团，也是一类重要的二氟甲基合成砌块. 常见化 合物主要包括: 二氟乙酸、二氟乙酸乙酯、二氟乙酸甲 酯、二氟乙酰卤等.

二氟乙酸的沸点相比于其常见的衍生物较高, 更加 便于储存. 但是由于其活性相对较低, 直接利用二氟乙 酸实现 3-(二氟甲基)吡坐合成的报道相对较少. 2013 年, 王宇小组 ${ }^{[14]}$ 报道了一条使用二氟乙酸 22 实现 3-(二氟甲 基)吡唑类化合物合成的通用路线. 作者首先利用丙炔 醇制得丙炔酸乙酯，随后与二甲胺通过加成反应实现氨 基丙烯酸酯 23 的合成. 接着在三光气的参与下，与二氟 乙酸反应制得含二氟甲基的双羰基中间体 24 , 最后通 过水合肼环化、硫酸二甲酯甲基化制得目标产物 3,4-二
取代 3-(二氟甲基)吡唑羧酸酯类化合物(Scheme 7). 该 方法总收率较高，区域选择性异构体比例较少，此外作 者很好的完成百克级的大量实验，体现了该方法优良的 应用价值。
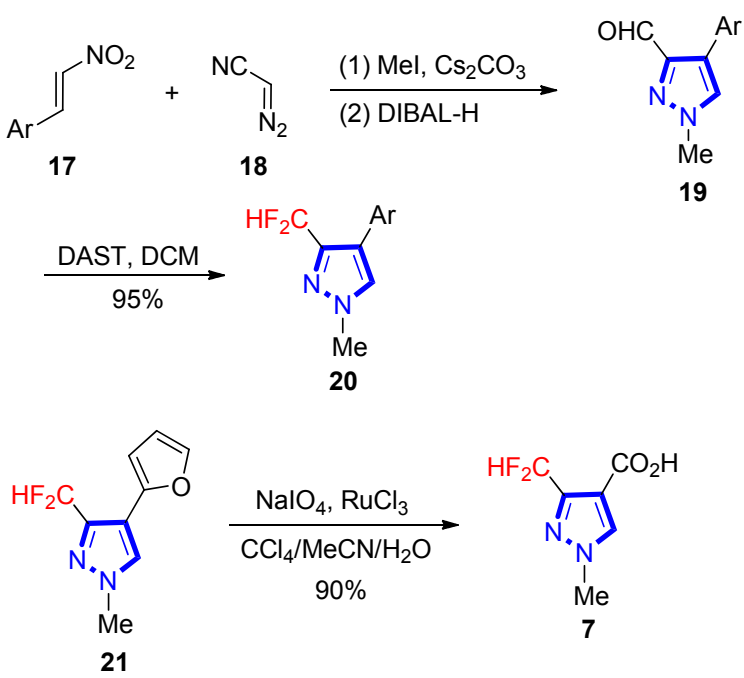

图式 6 以氟化试剂 DAST 作为氟源合成 3-(二氟甲基)吡唑-4羧酸

Scheme 6 Construction of 3- $\mathrm{CHF}_{2}$-pyrazole- $4-\mathrm{CO}_{2} \mathrm{H}$ by using DAST
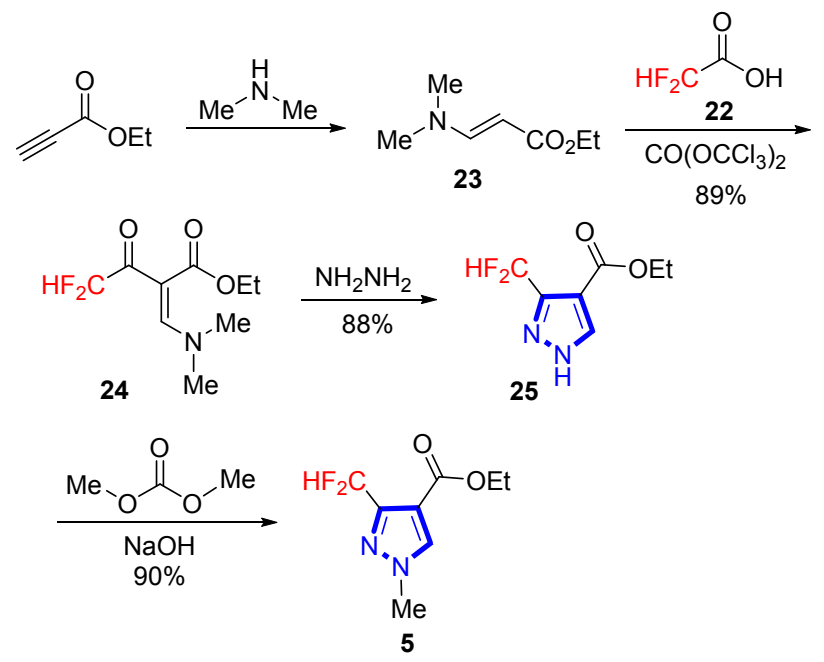

图式 7 以二氟乙酸作为氟源合成 3-(二氟甲基)吡唑-4-羧酸酯 Scheme 7 Construction of 3- $\mathrm{CHF}_{2}$-pyrazole-4-carboxylate by using $\mathrm{CF}_{2} \mathrm{HCO}_{2} \mathrm{H}$

二氟乙酸酯类化合物通常是由二氟乙烯 ${ }^{[15]}$ 二二氟乙 腈 ${ }^{[16]}$ 等含氟原料制得. 相比于二氟乙酸，二氟乙酸酯类 化合物活性更高，被大量地应用于 3-(二氟甲基)吡唑的 构建. 1995 年, Talley 小组 ${ }^{[17]}$ 利用二氟乙酸乙酯(26)合成 了含二氟甲基的 1,3 二酮类化合物 $\mathbf{2 8}$, 随后与芳基肼类 化合物环化制得 3,5-二取代-3-(二氟甲基)吡唑类化合物 29, 收率最高可达 93\% (Scheme 8a), 但是 1,3-二酮类化 
合物合成吡唑类化合物也存在区域选择性问题 ${ }^{[18]}$, 该 报道并未指出 3-(二氟甲基)吡唑类化合物所占的比例. 2005 年, Norris 小组 ${ }^{[19]}$ 同样使用 4,4-二氟-1-芳基-1,3-丁 二酮与芳基肼 31 反应制得 3-(二氟甲基)吡唑类化合物 (Scheme 8b). 作者研究发现以异丙醇作为溶剂, 不加催 化剂时, 该反应进行较慢, 需要反应 1 5 d, 而且 5-(二 氟甲基)吡唑 7 异构体较多, 比例可达 $25 \%$. 当在反应体 系中加入硫酸之后, 该反应只需 $1 \mathrm{~h}$ 即可完全反应, 此 外 5-(二氟甲基)吡唑占比降至 3\%.

(a)

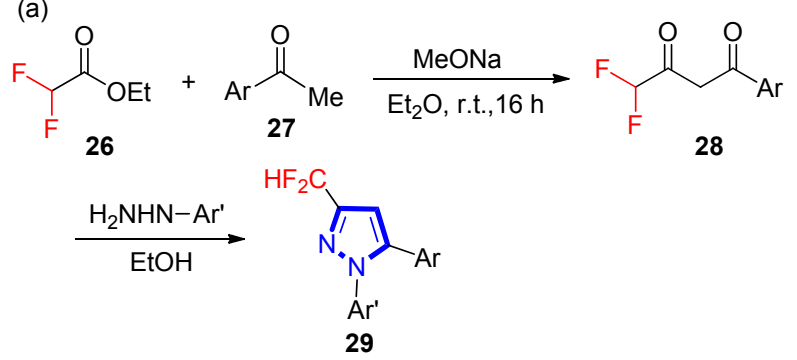

(b)
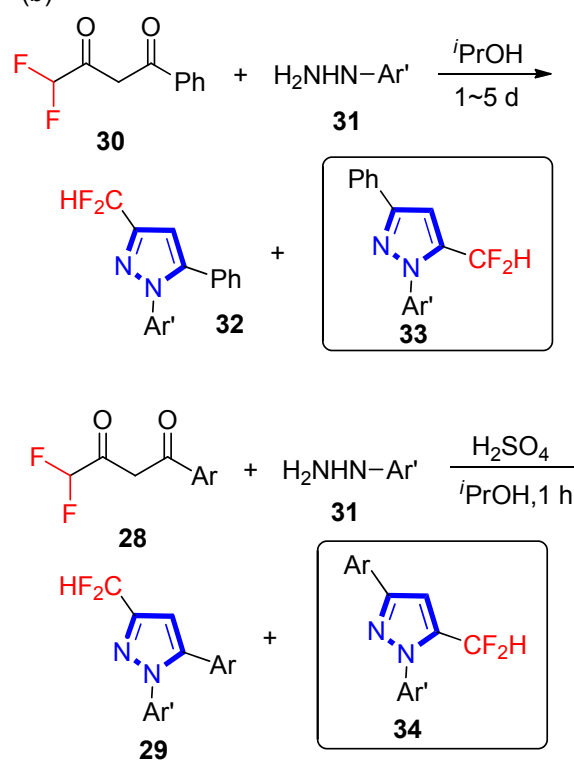

图式 8 通过含二氟甲基的 1,3 二酮类化合物实现二氟甲基吡 唑的构建

Scheme 8 Construction of $3-\mathrm{CHF}_{2}$-pyrazoles by $\mathrm{CHF}_{2}$-containing 1,3-diketone compounds

2006 年, Gosselin 小组 ${ }^{[20]}$ 使用 $10 \mathrm{~mol} \cdot \mathrm{L}^{-1}$ 盐酸催化 1,3-丁二酮类化合物 28 与芳基肼 $\mathbf{3 1}$ 反应, 制得了一系 列 3-(二氟甲基)吡唑类化合物 (Scheme 9). 该方法对 3-(二氟甲基)吡唑类化合物的区域选择性有着较为明显 的提高, 最高可达 $99.8 \%$. 但是上述几种方法得到产物 都是 3,5 位二取代吡唑 29, 并不能够直接构建出 3,4-二 取代 3-(二氟甲基)吡唑结构单元.

2008 年, 巴斯夫 Markus 小组 ${ }^{[21]}$ 以二氟乙酸酯类化 合物作为起始原料, 在碱性条件下, 与乙酸乙酯作用实

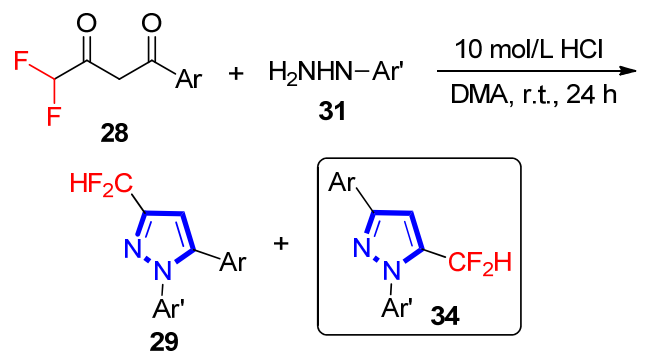

图式 9 盐酸催化实现 3-(二氟甲基)吡唑类化合物的构建 Scheme 9 Hydrochloric acid-catalyzed synthesis of 3- $\mathrm{CHF}_{2}$-pyrazoles

现二氟乙酰乙酸乙酯(36)的合成，接着在原甲酸三乙酯 的作用下，制得 2-(乙氧基亚甲基)-4,4-二氟-3-氧代丁酸 乙酯(37), 最后与甲基肼反应合成重要的母核结构 3-(二 氟甲基)吡唑-4-羧酸酯(Scheme 10). 该方法因其原料价 廉、路线简短易操作、收率较高, 在随后的合成研究中 被广泛地应用 ${ }^{[7,22,23]}$, 此外, 该路线也是目前工业上普 通采用的方法 ${ }^{[24]}$.
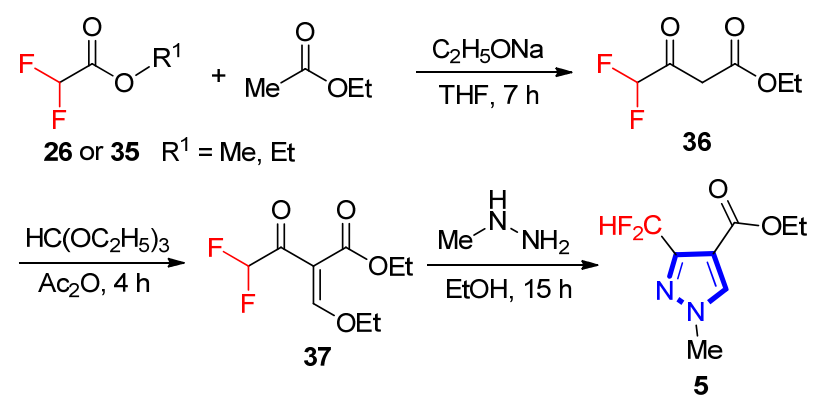

图式 10 以二氟乙酸酯类作为氟源合成 3-(二氟甲基)吡唑类 化合物

Scheme 10 Synthesis of 3- $\mathrm{CHF}_{2}$-pyrazoles by using difluoroacetates

同年该公司 Rack 等 ${ }^{[25]}$ 在上述工作的基础上, 利用 三氟甲基烯酮 38 的脱氟氢化反应，实现关键中间体 37 的合成，随后与甲基肼环化以较好的收率制得 3-(二氟 甲基)吡唑-4-羧酸酯类化合物. 使用该方法目标产物的 区域选择性较好，3-(二氟甲基)吡唑占比可达 94\% (Scheme 11).

与此同时，先正达公司 ${ }^{[7]}$ 报道以二氟甲基烯酮中间 体 37 作为起始原料，在甲基胺的作用下，生成乙烯基胺 类化合物 40, 随后加入氯胺和氢化钠, 制得 3,4-二取代3-(二氟甲基)吡唑羧酸酯类化合物(Scheme 12). 该方法 虽然合成路线短, 收率较高, 但是氯胺 $\left(\mathrm{NH}_{2} \mathrm{Cl}\right)$ 的现场 制备及严格的温度控制影响其实际应用 ${ }^{[26]}$.

2008 年, 先正达公司 ${ }^{[7]}$ 尝试以二氟乙酸乙酯(26)作 为底物，直接与甲基肼反应，制得二氟乙酰肼类化合物， 随后与丙炔酸酯通过环加成反应直接得到 3-(二氟甲基) 吡唑-4-羧酸酯(Scheme 13). 该方法虽然路线短, 但是文 
<smiles>CCOC=C(C(=O)OCC)C(=O)C(F)(F)F</smiles>

38<smiles>CCOC(=O)c1cn(S(C)(=O)=O)nc1C(=O)OCC</smiles>

图式 11 以二氟乙酸酯类作为氟源合成 3-(二氟甲基)吡唑类 化合物

Scheme 11 Synthesis of 3-CHF - -pyrazoles by using difluoroacetates<smiles>CCOC=C(C(=O)OCC)C(=O)C(F)F</smiles>

37

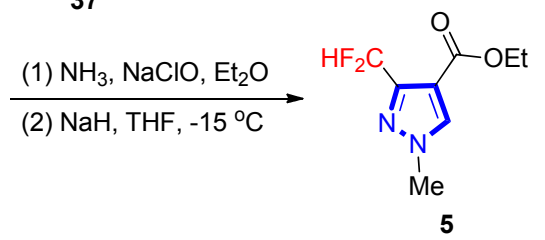

图式 12 利用二氟乙酸酯衍生物构建 3-(二氟甲基)吡唑类化 合物

Scheme 12 Construction of 3- $\mathrm{CHF}_{2}$-pyrazoles by using difluoroacetate derivatives

中并未给出反应的收率和区域选择性.

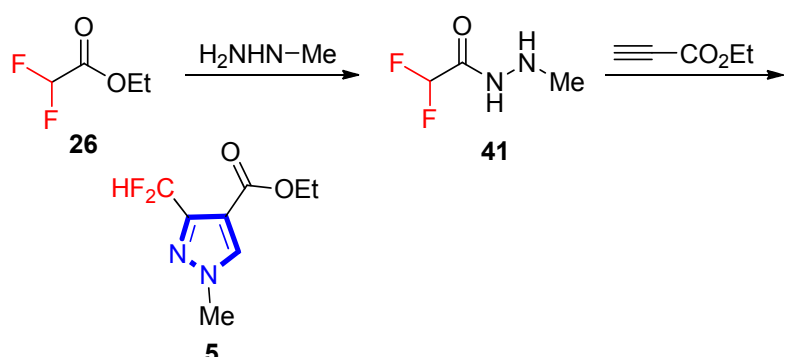

图式 13 以二氟乙酸乙酯为氟源合成 3-(二氟甲基)吡唑-4-羧 酸酯

Scheme 13 Synthesis of $3-\mathrm{CHF}_{2}$-pyrazoles by using ethyl difluoroacetate

2010 年, 该公司 Bowden 小组 ${ }^{[26]}$ 利用二氟乙酸甲酯 35 与水合肼反应制得二氟乙酰肼 41, 通过多聚甲醛与 钯碳的甲基化反应引入甲基, 随后与丙炔酸酯通过环化 反应得到目标产物(Scheme 14). 该反应虽然甲基化这 一步收率较低, 而且环化反应没有给出收率, 但是文中 提到可以拿到 3-(二氟甲基)吡唑.

2011 年, Dochnahl 小组 ${ }^{[23]}$ 以二氟甲基烯酮中间体 37 作为起始原料, 在对甲苯磺酸的催化下, 与苯甲醛衍生

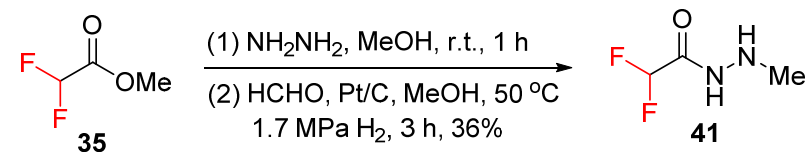<smiles>C#CC(OCC)OCCOC(=O)OCC</smiles>

图式 14 利用水合肼实现 3-(二氟甲基)吡唑类化合物的构建 Scheme 14 Construction of 3-(difluoromethyl)pyrazoles by methyl difluoroacetate

的腙 42 反应，以 $83 \%$ 的收率拿到 3-(二氟甲基)吡唑-4羧酸酯. 该方法虽然腙 $\mathbf{4 2}$ 制备较为复杂，但是在反应体 系中，作者并未检测到异构体的存在，具有良好的应用 价值(Scheme 15).

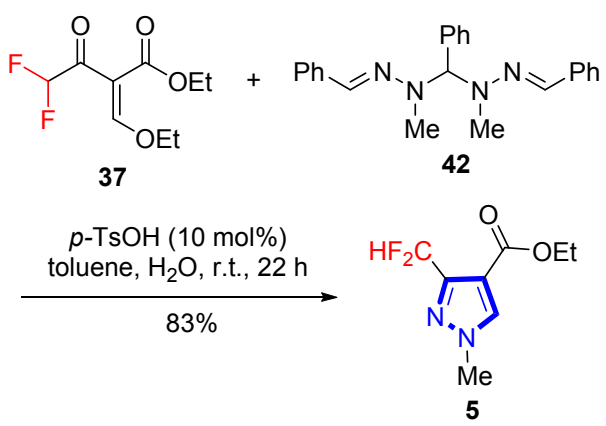

图式 15 利用二氟甲基烯酮实现 3-(二氟甲基)吡唑-4-羧酸酯 的构建

Scheme 15 Construction of 3- $\mathrm{CHF}_{2}$-pyrazole-4-carboxylate by using difluoroacetate derivatives

二氟氯甲基官能团也可以作为二氟甲基的来源，反 应后期通过还原脱氯即可得到二氟甲基官能团. 2011 年, Langer 课题组 ${ }^{[27]}$ 利用氯代二氟甲基羧酸酯衍生物 43 (或 44)与烷基肼作用，合成了一系列二氟氯甲基吡唑类 化合物 45. 随后在三丁基氢化锡和偶氮二异丁腈 (AIBN)的作用下还原氢化，制得 3-(二氟甲基)吡唑类化 合物 46. 该方法环化收率较高, 但是自由基脱氯反应收 率较低, 此外文中并未提到区域选择性的问题(Scheme 16).

2012 年，索尔维集团 Braun 小组 ${ }^{[28]}$ 同样使用二氟氯 甲基羧酸酯的衍生物 47 与甲基肼作用，制得 3-(氯二氟 甲基)吡唑羧酸酯(90). 随后在锌粉和氟化铯的作用下还 原氢化，制得 3,4-二取代-3-(二氟甲基)吡唑羧酸酯类化 合物(Scheme 17). 该方法虽然脱氯收率较高, 但是环化 的区域选择性较差，异构体 5-(二氟甲基)吡唑比例可达 $15 \%$.

2008 年, Sosnovskikh 小组 ${ }^{[29]}$ 以含二氟甲基的 1,3 二 酮类化合物 49 为底物, 在低温下与甲基肼作用, 合成了 


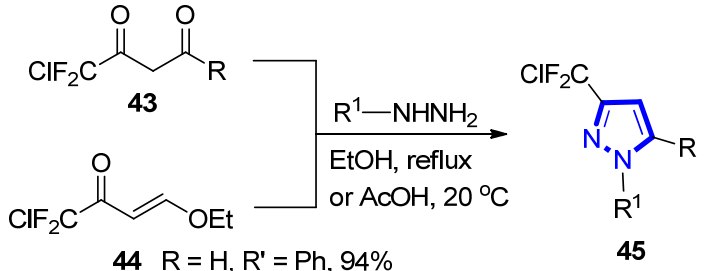

$44 \mathrm{R}=\mathrm{H}, \mathrm{R}^{\prime}=\mathrm{Ph}, 94 \%$

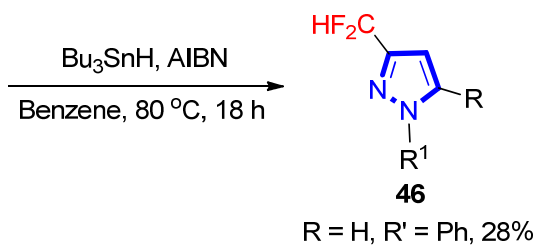

图式 16 利用二氟氯乙酸酯的衍生物构建 3-(二氟甲基)吡唑 类化合物

Scheme 16 Construction of 3- $\mathrm{CHF}_{2}$-pyrazoles by using difluorochloroacetate derivatives

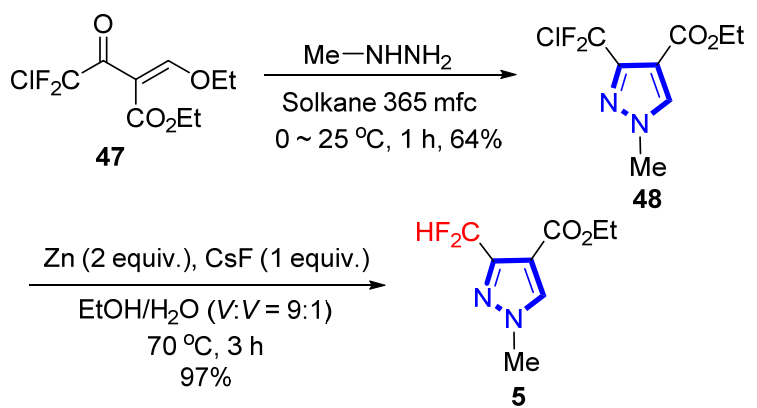

图式 17 利用二氟氯乙酸酯衍生物合成 3-(二氟甲基)吡唑类 化合物

Scheme 17 Construction of 3- $\mathrm{CHF}_{2}$-pyrazoles by using difluorochloroacetate derivatives

一系列 3,4-二取代-3-(二氟甲基)吡唑酮类化合物 $\mathbf{5 0}$ (Scheme 18). 该方法利用环状中间体很好的解决了异 构体 5-(二氟甲基)吡唑的产生，具有良好的应用价值.

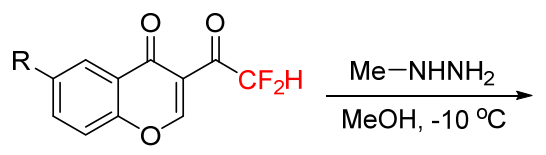

49

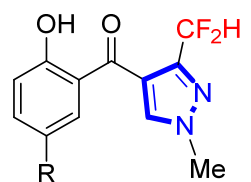

50

$$
\mathrm{R}=\mathrm{H}, 70 \% ; \mathrm{R}=\mathrm{Cl}, 74 \%
$$

图式 18 利用 1,3 二酮类化合物实现二氟甲基吡唑类化合物 合成

Scheme 18 Construction of 3- $\mathrm{CHF}_{2}$-pyrazoles by using 1,3-diketones

从上面的总结可以看出, 利用生成的 1,3 二羰基类 化合物与肼类化合物的环化反应是构建 3-(二氟甲基)吡 唑环的有效方法. 二氟乙酰卤也可以通过该方法实现 3,4-二取代-3-(二氟甲基)吡唑的构建.

2005 年, 拜尔农作物科学公司 ${ }^{[30]}$ 利用二氟乙酰卤 作为氟源, 与 $N, N$-二甲氨基丙烯酸酯(23)反应制得二氟 甲基双羰基中间体 24, 最后与甲基胇环化得到 3-(二氟
甲基)吡唑羧酸酯类化合物(Scheme 19a). 该方法路线 短, 收率高, 但是也存在一些问题. 首先反应条件较为 苛刻，其次第二步环化反应中 5-(二氟甲基)-吡唑异构体 比例较高, 可达 $9 \%$. 随后, 巴斯夫公司科研人员 ${ }^{[1]}$ 对于 该类合成方法进行补充，利用二氟乙酰氟(51)作为氟源， 与 3-(1-哌啶基)丙烯酸乙酯(52)反应制得二氟甲基双羰 基中间体 53, 随后与甲基肼环化得到 3-(二氟甲基)吡唑 羧酸酯类化合物(Scheme 19b). 该方法相比前一路线, 合成过程中异构体副产物明显减少(比例降至 $3 \%$ ), 反 应收率提高至 $97 \%$ ，表现出良好的应用价值. (a) Bayer<smiles>[X]C(=O)C(F)F</smiles>
$\mathrm{X}=\mathrm{Cl}, \mathrm{Br}, \mathrm{F}$
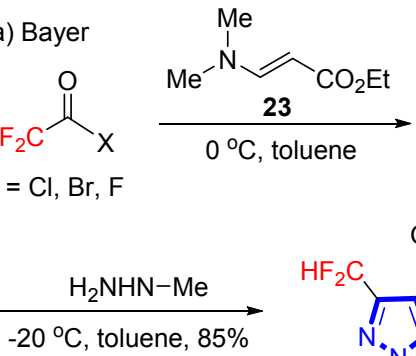<smiles>CCOC(=O)c1cn(C)nc1C(F)(F)F</smiles>

(b) BASF<smiles>O=C(F)C(F)(F)F</smiles><smiles>CCOC(=O)C=CN1CCCCC1</smiles>

51<smiles>CCOC(=O)c1cn(C)nc1C(F)(F)F</smiles>

5
53<smiles>CCOC(=O)C(=CN(C)C)C(=O)C(F)F</smiles><smiles>CCOC(=O)C(=CN1CCCCC1)C(=O)C(F)(F)F</smiles>

图式 19 以二氟乙酰卤作为氟源合成 3-(二氟甲基)吡唑-4-羧 酸酯

Scheme 19 Construction of 3- $\mathrm{CHF}_{2}$-pyrazole-4-carboxylate by using difluoroacetyl halide

2008 年, Zierke 小组 ${ }^{[32]}$ 利用二氟乙酰氯(54)与乙基 乙烯基醚反应，制得二氟甲基丙烯酮中间体 55, 随后直 接加入甲基肼环化制得 3-(二氟甲基)吡唑(6), 最后通过 $N$-溴代琥珀酰亚胺的澳代反应得到 4-溴-3-(二氟甲基)吡 唑化合物 57 (Scheme 20). 该方法虽然各步反应收率较 高，但是 5-(二氟甲基)吡唑(58)异构体比例较高，达到 $30 \%$.

2009 年, Pazenok 小组 ${ }^{[33]}$ 以二氟乙酰氯作为氟源, 与 $N, N$-二甲氨基丙烯酸酯反应制得二氟甲基双羰基中 间体 24, 与苯腙类化合物 59 通过双键的亲核取代反应, 制得二氟甲基腙类中间体 60, 随后在对甲苯磺酸的作 用下，自环化得到 3-(二氟甲基)吡唑-4-羧酸酯类化合物 (Scheme 21). 该方法很好地解决了反应区域选择性的 问题，以 90\%的收率得到单一构型的 3-(二氟甲基)吡唑, 
<smiles>CCOC=CC=CC(=O)C(=O)CC(CC(=O)C(F)F)OCC</smiles>

54

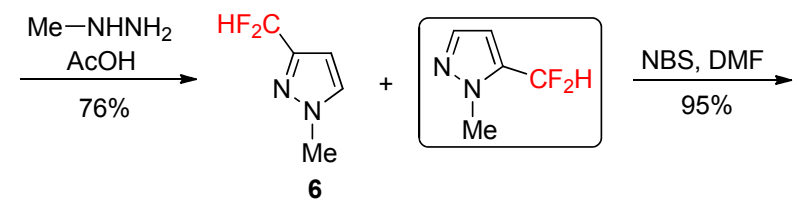<smiles></smiles>

图式 20 以二氟乙酰氯作为氟源合成 3-(二氟甲基)吡唑-4-羧 酸酯

Scheme 20 Construction of 3- $\mathrm{CHF}_{2}$-pyrazole-4-carboxylate by using difluoroacetyl chloride

具有良好的应用价值.

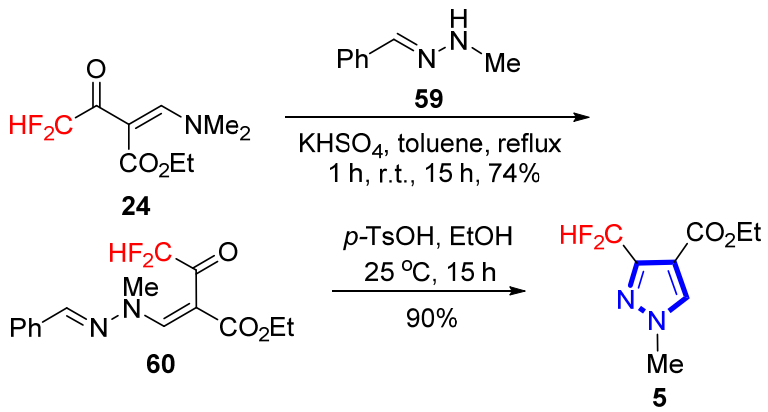

图式 21 以二氟乙酰氯的衍生物为底物合成 3-(二氟甲基)吡 唑-4-羧酸酯

Scheme 21 Synthesis of 3- $\mathrm{CHF}_{2}$-pyrazole-4-carboxylate with derivatives of difluoroacetyl chloride

2016 年, 王明春小组 ${ }^{[34]}$ 利用腙类化合物与炔酸酯 作用, 生成氨基丙烯酸酯类化合物 61, 以二氟乙酰氯作 为氟源，反应得到二氟甲基腙类中间体 60. 随后在盐酸 的作用下环化制得 3-(二氟甲基)吡唑-4-羧酸酯类化合物 (Scheme 22). 与 Pazenok 小组路线相比, 该路线在反应 后期引入二氟甲基, 减少氟损失. 此外, 本方法也以较 高的收率得到单一构型的目标产物.

\section{3 以含氟重氮类化合物作为氟砌块}

含氟重氮也是一种重要的含氟砌块, 含氟重氮主要 是通过含氟胺类化合物的重氮化反应制得, 早在 20 世 纪 40 年代已有相关报道 ${ }^{[35]}$, 常被用于各种含氟杂环的 构建 ${ }^{[36]}$

2015 年, 乌克兰 Mykhailiuk 课题组 ${ }^{[37]}$ 使用二氟乙 胺、乙酸、亚硝酸叔丁酯在氯仿中加热回流合成了二氟 重氮乙烷 $(63)$, 随后直接在体系中加入缺电子炔烃, 通 过[3+2]环加成反应合成了一系列 3-(二氟甲基)吡唑类
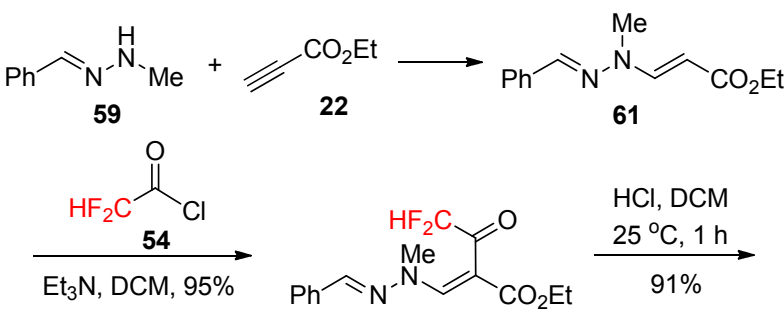

60<smiles>[Y]n1cc(C(=O)OCC)c(CF)n1</smiles>

图式 22 以二氟乙酰氯作为氟源合成 3-(二氟甲基)吡唑-4-羧 酸酯

Scheme 22 Construction of 3- $\mathrm{CHF}_{2}$-pyrazole-4-carboxylate by using difluoroacetyl chloride

化合物(Scheme 23). 这种一锅法合成 3-(二氟甲基)吡唑 不需要使用催化剂, 也不需要分离危险试剂, 一经报道 就引起了广泛的关注.

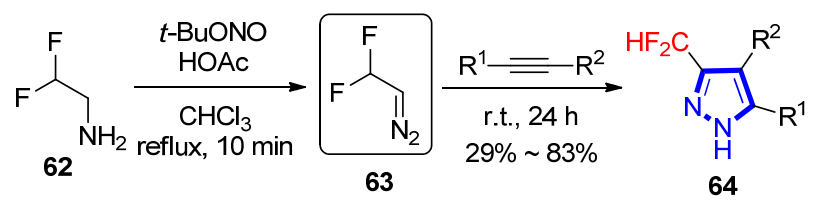

图式 23 二氟重氮用于 3-(二氟甲基)吡唑类化合物合成 Scheme 23 Construction of $3-\mathrm{CHF}_{2}$-pyrazoles by using $\mathrm{CHF}_{2-}$ $\mathrm{CHN}_{2}$

2016 年, Koenigs 小组 ${ }^{[38]}$ 把流动相技术应用于 3-(二 氟甲基)吡唑类化合物的合成(Scheme 24). 该技术的应 用使得二氟重氮乙烷的合成相对安全，操作简便，此外 收率也得到相应的提高，最高可达 99\%.

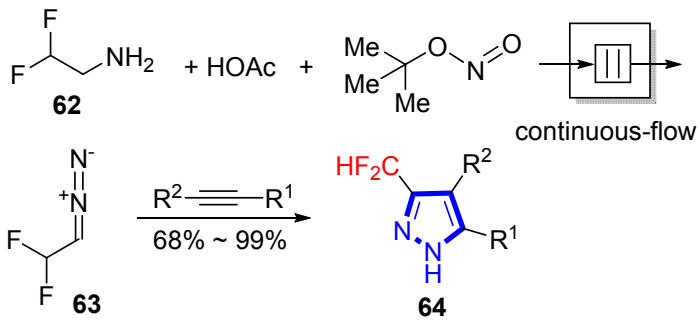

图式 24 流动相技术应用于 3-(二氟甲基)吡唑类化合物的合 成

Scheme 24 Construction of $3-\mathrm{CHF}_{2}$-pyrazoles by using the technology of continuous flow synthesis

2017 年, Mykhailiuk 小组 ${ }^{[39]}$ 利用二氟重氮乙烷与缺 电子烯烃的 $[3+2]$ 环加成反应，合成了一系列二氟甲基 吡唑啉类化合物 66. 该类化合物在加热条件下经二氧 化锰氧化得到 3-(二氟甲基)吡唑类化合物(Scheme 25). 


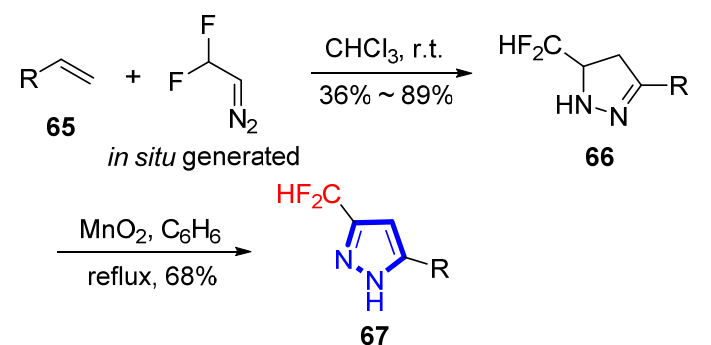

图式 25 二氟重氮用于 3-(二氟甲基)吡唑类化合物的合成 Scheme 25 Construction of $3-\mathrm{CHF}_{2}$-pyrazoles by using $\mathrm{CHF}_{2}$ $\mathrm{CHN}_{2}$

同年, Jamison 小组 ${ }^{[40]}$ 使用流动相技术, 以二氟重氮 乙烷为氟源成功得到联苯吡菌胺农药类似物 (Scheme 26). 作者首先利用二氟乙胺通过流动相技术制得二氟 重氮乙烷, 随后在体系中通入三甲基硅基丙炔酸酯, 制 得多取代的 3-(二氟甲基)吡唑 68. 在四丁基氟化铵 (TBAF)的作用下脱除三甲基硅基, 最后通过碘甲烷的 甲基化反应制得 3-(二氟甲基)吡唑-4-羧酸酯类化合物. 上述报道 3,4-二取代-3-(二氟甲基)吡唑类化合物的构建 都是基于关键中间体二氟重氮乙烷，但是二氟重氮乙烷 是一种不稳定的有毒气体, 难以储存和大量使用的特 性，对其进一步的开发应用有一定的限制.

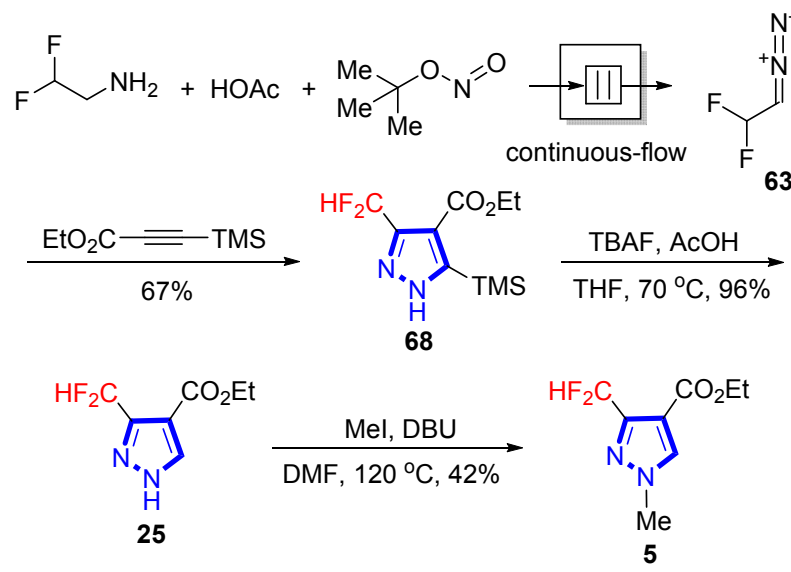

图式 26 利用二氟重氮合成 3-(二氟甲基)吡唑-4-羧酸酯类化 合物

Scheme 26 Construction of 3- $\mathrm{CHF}_{2}$-pyrazole-4-carboxylate by using $\mathrm{CHF}_{2} \mathrm{CHN}_{2}$

2018 年, 马军安小组 ${ }^{[41]}$ 开发出一种新型二氟重氮 试剂一苯砜基二氟重氮乙烷 69, 作者利用含氟砌块与乙 烯基砜 70 反应，可制得单取代的含氟吡唑类化合物 71. 随后经过甲基化、脱除砜基保护和溴代制得 4-溴-1-甲 基-3-(二氟甲基)吡唑(57) (Scheme 27). 利用重氮试剂苯 砜基二氟重氮乙烷可以区域选择性的构建出 3,4-二取 代-3-(二氟甲基)吡唑类化合物, 不会产生异构体. 此外, 含氟重氮试剂在室温条件下为稳定的黄色液体, 便于储 存使用.

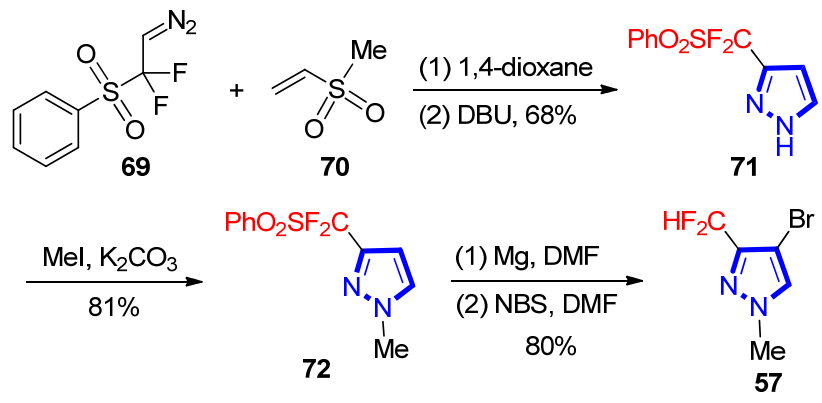

图式 27 利用苯砜基二氟重氮乙烷合成 3-(二氟甲基)吡唑类 化合物

Scheme 27 Construction of 3- $\mathrm{CHF}_{2}$-pyrazoles by using $\mathrm{PhSO}_{2}-$ $\mathrm{CF}_{2} \mathrm{CHN}_{2}$

\section{4 其它含氟砌块}

二氟甲基炔酮类化合物也常被用于构建含二氟甲 基环状化合物. 1989 年 Linderman 小组 ${ }^{[42]}$ 利用二氟甲基 丙炔酮(73)与水合肼反应，实现了 3-(二氟甲基)吡唑类 化合物的构建(Scheme 28). 作者认为该反应机理可能 为: 首先通过肼对炔烃的迈克尔环加成、或者通过肼对 酮羰基的加成形成中间体，随后通过芳构化反应实现 3-(二氟甲基)吡唑的高区域选择性合成.

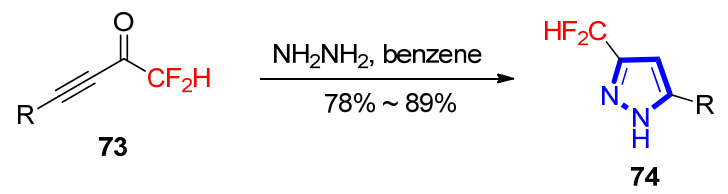

图式 28 利用二氟甲基炔酮实现二氟甲基吡唑的选择性合成 Scheme 28 Construction of $3-\mathrm{CHF}_{2}$-pyrazoles by using difluoromethylalkyne

1990 年, Hamper 小组 ${ }^{[43]}$ 在上述工作的基础上，以二 氟甲基丙炔酸酯(75)作为氟源，与甲基肼作用制得含羟 基的 3-(二氟甲基)吡唑类化合物(Scheme 29). 该反应虽 然条件温和，操作简便，但是反应收率较低，原料制备 较困难.

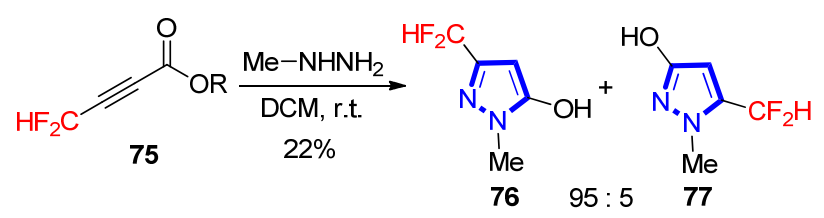

图式 29 利用二氟甲基炔酸酯实现二氟甲基吡唑类化合物的 合成

Scheme 29 Synthesis of 3-CHF 2 -pyrazoles with difluoromethylalkynate

2008 年, 先正达公司 ${ }^{[7]}$ 以 1,1 -二氟丙酮 78 作为起始 原料，与甲基肼反应制得二氟甲基腙类中间体 79, 在三 氯氧磷的催化下，环化制得 3-(二氟甲基)吡唑醛类化合 物，最后经过双氧水的氧化实现 3-(二氟甲基)吡唑羧酸 
类化合物的合成(Scheme 30). 该方法虽然路线短, 操作 简便，但是 1,1-二氟丙酮价格昂贵 (1 g/100 RMB), 反应 收率较低, 且异构体含量较高分离困难, 需要进一步的 优化.

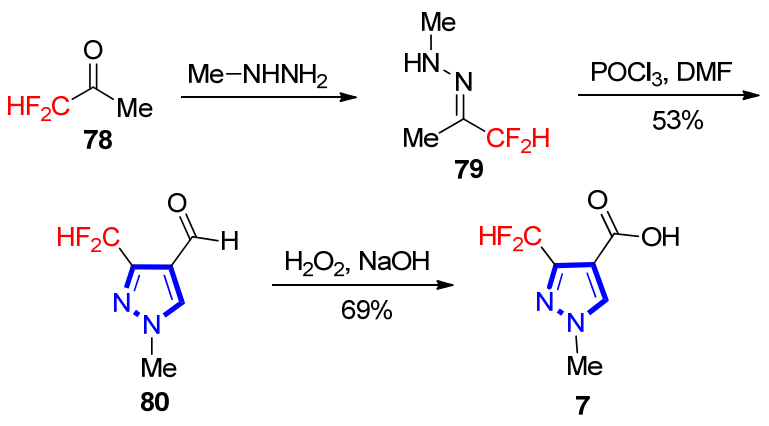

图式 30 以 1,1-二氟丙酮作为氟源合成 3-(二氟甲基)吡唑-4羧酸

Scheme 30 Construction of 3- $\mathrm{CHF}_{2}$-pyrazole-4- $\mathrm{CO}_{2} \mathrm{H}$ by using 1,1-difluoroacetone

四氟乙基二甲胺(TFEDMA), 因其可提供二氟甲基 官能团，也常被用做二氟甲基合成砌块 ${ }^{[44]}$. TFEDMA 主 要是通过廉价的四氟乙烯和二甲胺在低温条件下制 得 ${ }^{[44 a]} .2008$ 年, Nett 小组 ${ }^{[45]}$ 利用 TFEDMA 与甲氧基丙烯 酸甲酯 82 反应制得 Vinamidinium 盐 83 (1,5-二氮杂戊二 烯盐), 随后在甲基肼的作用下环化制得 3-(二氟甲基)吡 唑-4-羧酸酯(Scheme 31a). 该反应路线较短, 而且可以 拿到中等的收率, 但是异构体 5-(二氟甲基)吡唑较多 $(13 \%)$. 与此同时, 拜尔公司 Pazenok 小组 ${ }^{[46]}$ 以甲氧基丙 烯酸乙酯 (86) 作为起始原料, 并延长第二步反应时间, 以 86\%总收率, $92: 8$ 的区域选择性制得 3-(二氟甲基) 吡唑-4-羧酸酯类化合物(Scheme 31b). 2009 年该小组 ${ }^{[32]}$ 利用丙烯酸酯的衍生物 89 代替上述反应过程中的甲氧 基丙烯酸酯和甲基肼, 直接与三氟乙基二甲胺硼酸盐 (88) 反应以 94\%收率制得 3-(二氟甲基)吡唑类化合物 (Scheme 31c). 在反应过程中, 作者并未检测到异构体 的产生, 此外该方法的路线短, 收率较高, 具有良好的 工业应用价值.

2010 年, 巴斯夫 Nett 小组 ${ }^{[4]}$ 再次使用四氟乙基二 甲胺作为氟源合成 3,4-二取代-3-(二氟甲基)吡唑类化合 物, 与上述方法不同的是, 在合成 Vinamidinium 盐过程 中加入了吡啶作为催化剂, 但是反应收率和异构体的控 制并没有进一步的提高(Scheme 32).

\section{2 总结与展望}

得益于 3,4-二取代-3-(二氟甲基)吡唑结构单元在医 药领域的大量应用，3,4-二取代-3-(二氟甲基)吡唑类化 合物的合成近年来取得一系列重要的进展. 本文主要从
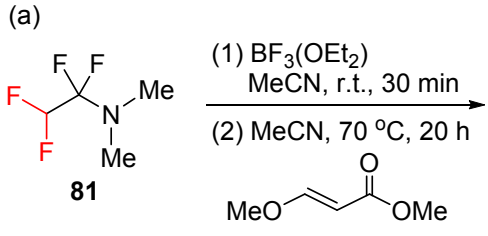<smiles>COC=C(C(=O)OC)C(=[N+](C)C)C(F)(F)Br</smiles>

82<smiles>COC(=O)c1cn(C)nc1C(=O)OC</smiles>

(b)<smiles>CN(C)C(F)(F)C(F)F</smiles><smiles>CCOC(=O)/C=C/OC</smiles><smiles>CCOC(=O)C(=COC)C(=[N+](C)Br)C(F)(F)F</smiles>

86

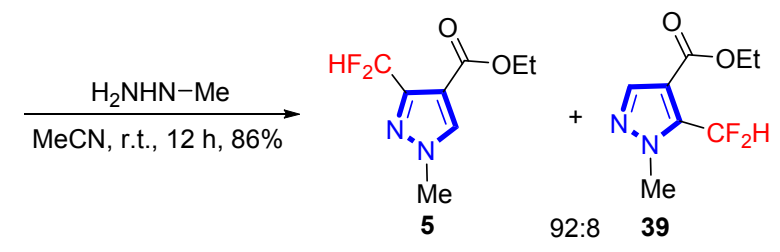

(c)

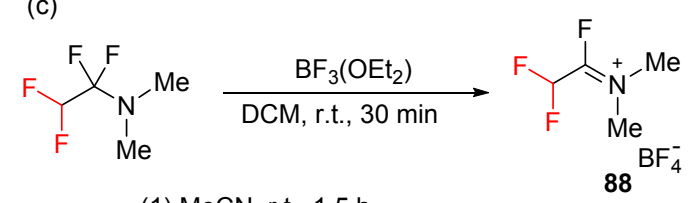

(1) $\mathrm{MeCN}$, r.t., $1.5 \mathrm{~h}$ $88^{\mathrm{BF}_{4}}$<smiles>CCOC(=O)/C=C/N(C)N=C(C)C</smiles>

图式 31 以 TFEDMA 为原料合成 3-(二氟甲基)吡唑-4-羧酸酯 Scheme 31 Construction of 3- $\mathrm{CHF}_{2}$-pyrazole-4-carboxylic acid by using TFEDMA

四个方面简要介绍了 3-(二氟甲基)吡唑类化合物合成的 方法. 从中可以看出, 二氟乙酸及其衍生物在 3-(二氟甲 基)吡唑类化合物合成中占据重要的地位, 利用含氟重 氮作为含氟砌块, 因其无异构体产生、较少添加剂的使 用, 也表现出优良性能. 目前合成 3,4-二取代-3-(二氟甲 基)吡唑类化合物的方法已经相对成熟但仍有不足，例 如: 利用含氟酯、含氟酰卤、TFEDMA 等氟源合成过程 中异构化和收率的问题; 以氟化试剂作为氟源, 位点选 择性以及收率的问题等. 因此发展高效、专一性、价廉 的方法实现 3,4-二取代-3-(二氟甲基)吡唑类化合物的合 成仍具有重大的研究价值. 


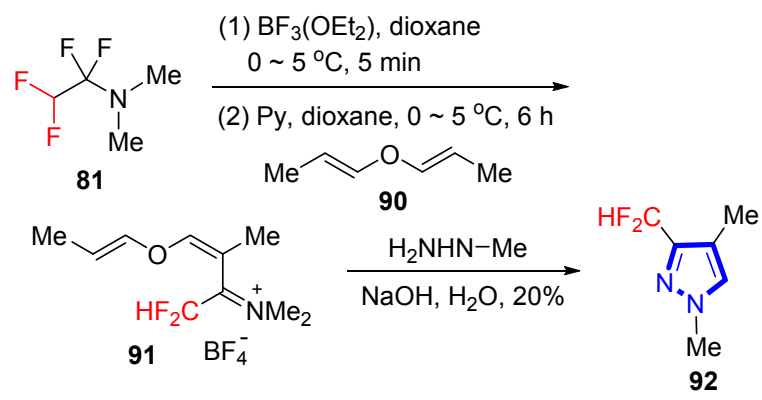

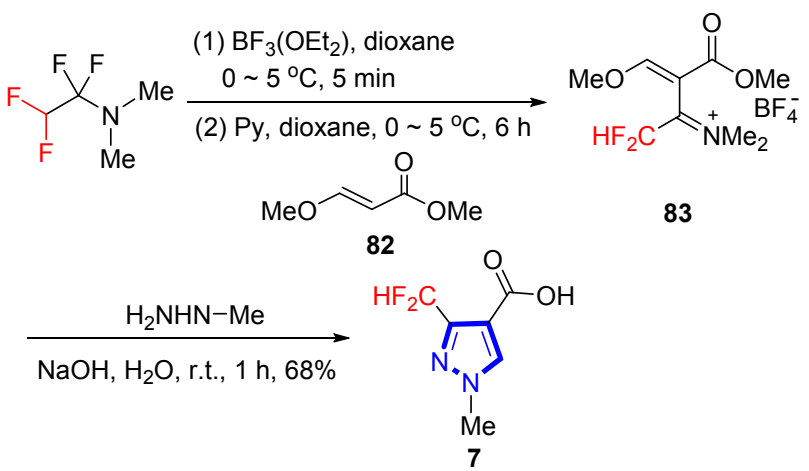

图式 32 以四氟乙基二甲胺作为氟源合成 3-(二氟甲基)吡唑 类化合物

Scheme 32 Construction of $3-\mathrm{CHF}_{2}$-pyrazoles by using TFEDMA

\section{References}

[1] (a) Yossi, Z.; Gali, S. M.; Dina, Y.; Anat, B.; Dafna, A.; Daniele, M.; Shlomi, E.; Shahaf, K.; Nissan, A.; Moran, M.; Eytan, G.; Sigal, S. J. Med. Chem. 2019, 62, 5628.

(b) Dai, J.-L.; Lei, W.-L.; Liu, Q. Acta Chim. Sinica 2019, 77, 911 (in Chinese).

(戴建玲, 雷文龙, 刘强, 化学学报, 2019, 77, 911.)

(c) Fu, X.-P.; Xiao, Y.-L.; Zhang, X.-G. Chin. J. Chem. 2018, 36, 143.

(d) Damian, E. Y.; Sebastian, B. V.; Postigo. A. Chem.-Eur. J. 2017, 23, 14676.

(e) Ni, C.-F.; Zhu, L.-G.; Hu, J.-B. Acta Chim. Sinica 2015, 73, 90 (in Chinese).

(倪传法, 朱林桂, 胡金波, 化学学报, 2015, 73, 90.)

(f) Yang, Y.; You, Z.; Qing, F. Acta Chim. Sinica 2012, 70, 2323 (in Chinese).

(杨义, 游正伟, 卿风翎, 化学学报, 2012, 70, 2323.)

(g) O'Hagan, D.; Wang, Y.; Skibinski, M.; Slawin, A. M. Z. Pure Appl. Chem. 2012, 84, 1587.

(h) Meanwell, N. A. J. Med.Chem. 2011, 54, 2529.

(i) Erichson, J. A.; McLoughlin, J. I. J. Org. Chem. 1995, 60, 1626.

[2] Mcloughlin, J. I.; Louis, St.; Metz, S. C. US 2005223526, 1992.

[3] (a) Zierke, T.; Maywald, V.; Rack, M.; Smidt, S. P.; Keil, M.; Wolf, B.; Koradin, C. WO 2009133179, 2009.

(b) Penning, T. D.; Talley, J. J.; Bertenshaw, S. R.; Carter, J. S.; Collins, P. W.; Doctor, S.; Greveto, M. J.; Lee, L. F.; Malecha, J. W.; Miyashiro, J. M.; Rogers R. S.; Rogier, D. J.; Yu, S. S.; Anderson, G. D.; Burton, E. G.; Gregory, S. A.; Icoboldt, C. M.; Perkus, W. E.; Seibert, K. A.; Veenhuizen, W.; Zhang, Y. Y.; Isakson, P. C. J. Med. Chem. 1997, 40, 1347.

(c) Liu, X.-H.; Zhao, W.; Shen, Z.-H.; Xing, J.-H.; Xu, T.-M.; Peng, W.-L. Eur. J. Med. Chem. 2017, 125, 881.

[4] (a) Qiu, S.-S.; Bai, Y.-L. Modern Agrochem. 2014, 6, 1 (in Chinese).

(仇是胜, 柏亚罗, 现代农药, 2014, 6, 1.) (b) Qiu, S.-S.; Bai, Y.-L. Modern Agrochem. 2015, 14, 1 (in Chinese). (仇是胜，柏亚罗，现代农药, 2015, 14, 1.)

[5] Xiao, H. World Pestic. 2007, 39, 12 (in Chinese). (䈗禾，世界农药, 2007, 39, 12.)

[6] Lantzsch, R.; Pazenok, S.; Memmel, F. WO 2005044804, 2005.

[7] Syngenta Participations AG EP 2008996, 2008.

[8] Kremsner, J. M.; Rack, M.; Pilger, C.; Kappe, C. O. Tetrahedron Lett. 2009, 50, 3665.

[9] Bolea, C.; Celanire, S.; Boudou, C.; Tang, L.; Rocher, J. P.; Liverton, N. J. WO 2012009009,2012

[10] Fan, X.-B.; Lin, X.-J.; Xu, X.-M.; Huang, C.; Shen, Q.-F. $C N$ $104016920,2014$.

[11] Sakamoto, R.; Kashiwagi, H.; Maruoka, K. Org. Lett. 2017, 19, 5126.

[12] Zhang, Y.; Chen, Z.; Nie, J.; Zhang, F.-G.; Ma, J.-A. J. Org. Chem. 2019, 84, 7148.

[13] (a) Liu, C.-B.; Meng, W.; Li, F.; Wang, S.; Nie, J.; Ma, J.-A. Angew. Chem., Int. Ed. 2012, 51, 6227.

(b) Wang, S.; Nie, J.; Zheng, Y.; Ma, J.-A. Org. Lett. 2014, 16, 1606.

(c) Zhang, F.-G.; Wei, Y.; Yi, Y. P.; Ma, J.-A. Org. Lett. 2014, 16, 3122 .

(d) Chen, Z.; Zheng, Y.; Ma, J.-A. Angew. Chem., Int. Ed. 2017, 56, 4569.

[14] Li, L.-F.; Xu, G. Y.; Zhao, D.-J.; Chen, M.; Wang, Y. Fine Chem. Intermed. 2011, 43(6), 17.

[15] Oharu, K.; Kumai, S. EP 0694523, 1995.

[16] Nishimiya, T.; Fuku, A.; Okamoto, S. WO 2008078479, 2008.

[17] Talley, J. J.; Penning, T. D.; Collins, P. W.; Rogier, D. J.; Malecha, J. W.; Miyachiro, J. M.; Bertenshaw, S. R.; Khanna, I. K.; Granets, M. J.; Rogers, R. S.; Carter, J. S.; Docter, S. H.; Yu, S. S. WO 9515316, 1995.

[18] (a) Singh, S. P.; Kumar, D.; Batra, H.; Naithani, R.; Rozas, I.; Elguero, J. Can. J. Chem. 2000, 78, 1109.

(b) Sloop, J. C. Bumgardner, C. L.; Loehle, W. D. J. Fluorine Chem. 2002, 118, 135.

[19] Norris, T.; Colon-Cruz, R.; Ripin, D. H. B. Org. Biomol. Chem. 2005, 3, 1844.

[20] Gosselin, F.; O'Shea, P. D.; Webster, R. A.; Reamer, R. A.; Tillyer, R. D.; Grabowski, E. J. J. Synlett 2006, 19, 3267.

[21] Gewehr, M.; Muller, B.; Grote, T.; Grammenos, W.; Schwogler, A.; Rheinheimer, J.; Blettner, G.; Schafer, P.; Schieweck, F.; Werner, F.; Rether, J.; Strathmann, S.; Stierl, R.; Scherer, M. WO 2005123690, 2005.

[22] (a) Wu, Z.-B.; Hu, D.-Y.; Kuang, J.-Q.; Cai, H.; Wu, S. X.; Xue, W. Molecules 2012, 17, 14205.

(b) Sun, J.-L.; Zhou, Y.-M. Molecules 2015, 20, 4383.

(c) Liu, X.-H.; Zhao, W.; Shen, Z.-H.; Xing, J.-H.; Xu, T.-M.; Peng, W.-L. Eur. J. Med. Chem. 2017, 125, 881.

(d) Qiao, L.; Zhai, Z.-W.; Cai, P.-P.; Tan, C.-X.; Weng, J.-Q.; Han, L.; Liu, X.-H.; Zhang Y.-G. J. Heterocycl. Chem. 2019, 56, 2536.

[23] Dochnahl, M.; Keil, M.; Gotz, R. WO 2011054733, 2010.

[24] Huang, X.-Y.; Shang, Y.; Wang, L.-P.; Wang, W. Agrochemicals 2018, 57(10), 703 .

[25] Rack, M.; Smidt, S. P.; Lohr, S.; Keil, M.; Dietz, J.; Rheinheimer, J.; Grote, T.; Zierke, T.; Lohmann, J. K.; Sukopp, M. WO 2008053043, 2008

[26] Bowden, M.; Gott, B. D.; Jackson, D. A. WO 2009000442, 2009.

[27] Iaroshenko, V. O.; Specowius, V.; Vlach, K.; Vilches-Herrera, M.; Ostrovskyi, D.; Mkrtchyan, S.; Villinger, A.; Langer, P. Tetrahedron 2011, 67, 5663.

[28] Braun, M. J.; Jaunzems, J. WO 2012010692, 2012.

[29] Sosnovskikh, V. Y.; Irgashev, R. A. Moshkin, V. S. Kodess, M. I. Russ. Chem. Bull. 2008, 57, 2146.

[30] Lantzsch, R.; Wolfgang, J.; Pazenok, S. WO 2005042468, 2004.

[31] Zierke, T.; Maywald, V.; Rack, M.; Smidt, S. P.; Keil, M.; Wolf, B.; Koradin, C. US 20110040096, 2009. 
[32] Zierke, T.; Rheinheimer, J.; Rack, M.; Smidt, S. P.; Altenhoff, A. G.; Schmidt-Leithoff, J.; Challand, N. WO $2008145740,2008$.

[33] Pazenok, S.; Lui, N.; Heinrich, J. D.; Wollner, T. WO 2009106230, 2009.

[34] Wang, M.-C.; Li, Q.-Y.; Luo, Z.-B. CN 107663172, 2016.

[35] Gilman, H.; Jones, R. G. J. Am. Chem. Soc. 1943, 65, 1458.

[36] (a) Morandi, B.; Carreira, E. M. Angew. Chem., Int. Ed. 2011, 50, 9085

(b) Morandi, B.; Carreira, E. M. Org. Lett. 2011, 13, 5984.

(c) Morandi, B.; Carreira, E. M. Angew. Chem., Int. Ed. 2010, 49, 938.

(d) Artamonov, O. S.; Mykhailiuk, P. K.; Voievoda, N. M.; Volochnyuk, D. M.; Komarov, I. V. Synthesis 2010, 443.

(e) Li, F.; Nie, J.; Sun, L.; Ma, J.-A. Angew. Chem., Int. Ed. 2013, $52,6255$.

(f) Peng, X.; Xiao, M.-Y.; Zeng, J.-L.; Zhang, F.-G.; Ma, J.-A. Org. Lett. 2019, 21, 4808 .

(g) Zhang, Z.-Q.; Zheng, M.-M.; Xue, X.-S.; Marek, I.; Zhang, F.-G.; Ma, J.-A. Angew Chem., Int. Ed. 2019, 58, 18191.

[37] Mykhailiuk, P. K. Angew Chem., Int. Ed. 2015, 54, 6558.

[38] Mertens, L.; Hock, K. J.; Koenigs, R. M. Chem.-Eur. J. 2016, 22, 9542.

[39] Li, J.; Yu, X.-L.; Cossy, J.; Lv, S.-Y.; Mykhailiuk, P. K.; Wu, Y. Eur.
J. Org. Chem. 2017, 266.

[40] Britton, J.; Jamison, T. F. Angew. Chem., Int. Ed. 2017, 56, 8823.

[41] Zeng, J.-L.; Chen, Z.; Zhang, F.-G.; Ma, J.-A. Org. Lett. 2018, 20 , 4562.

[42] Linderman, R. J.; Kirollos, K. S. Tetrahedron Lett. 1989, 30, 2049.

[43] (a) Hamper, B. C. J. Fluorine Chem. 1990, 48, 123.

(b) Hamper, B. C.; Kurtzweil, M. L.; Beck, J. P. J. Org. Chem. 1992, 57,5680 .

[44] (a) England, D. C.; Melby, L. R.; Dietrich, M. A.; Lindsey, R. V. J. Am. Chem. Soc. 1960, 82(19), 5116.

(b) Wakselman, C. Tordeux, M. J. Chem. Soc., Chem. Commun. 1975, 956.

(c) Sergiy P.; Florence G.; Grégory L.; Norbert L.; Jean-Pierre, V;; Frédéric, R. L. Eur. J. Org. Chem. 2013, 4249.

(d) Etienne, S.; Baptiste, R.; Armen, P.; Jean-Pierre, V.; Sergii, P.; Frédéric, R. L. Org. Lett. 2015, 17(18), 4510.

(e) Etienne, S. Grégory, L.; Jean-Pierre, V.; Norbert, L.; Sergiy, P.; Frédéric, R. L. Eur. J. Org. Chem. 2018, 3792.

[45] Nett, M.; Grote, T.; Lohmann, J. K.; Dietz, J.; Smidt, S. P.; Rack, M.; Zierke, T. WO 2008152138, 2008.

[46] Pazenok, S.; Lui, N.; Neeff, A. WO 2008022777, 2008.

[47] Nett, M.; Grote, T.; Lohmann, J. K.; Dietz, J.; Smidt, S. P.; Rack, M.; Zierke, T. US 2010084994, 2010 